\title{
Non-neoplastic portal vein thrombosis in HCV cirrhosis: the weight of inflammation on a fragile hemostatic balance
}

\author{
Niccolò Bitto', Vincenzo La Mura, ${ }^{1,2}$ \\ ${ }^{1}$ Unit of Internal Medicine, IRCCS San Donato, University of Milan, San Donato Milanese (MI), Milan, Italy; ${ }^{2}$ Centro di Ricerca Coordinata A. M. e A. \\ Migliavacca, per lo Studio e la Cura delle Malattie del Fegato, Milano, Italy \\ Correspondence to: Vincenzo La Mura. Unit of Internal Medicine, IRCCS San Donato, University of Milan, San Donato Milanese (MI), Via Morandi, \\ Milan 30 20097, Italy. Email: vincenzo.lamura@unimi.it. \\ Provenance: This is a guest Editorial commissioned by Associate Editor-in-Chief Xingshun Qi (Department of Gastroenterology, General Hospital of \\ Shenyang Military Area, Shenyang, China). \\ Comment on: Abu El-Makarem MA, El-Akad AF, Elian MM, et al. Non-neoplastic Portal Vein Thrombosis in HCV Cirrhosis Patients: Is it an \\ Immuno-Inflammatory Disorder? Ann Hepatol 2017;16:574-83.
}

Received: 29 August 2017; Accepted: 14 September 2017; Published: 11 October 2017.

doi: $10.21037 /$ amj.2017.09.12

View this article at: http://dx.doi.org/10.21037/amj.2017.09.12

The historical paradigm of spontaneous bleeding tendency of cirrhosis as consequence of an intrinsic coagulopathy has faded in the last years (1). This theory has been first challenged by milestones studies of Tripodi et al. demonstrating normal thrombin generation by plasma from cirrhotic patients and, consequently, the need of more comprehensive coagulation tests to evaluate hemostatic changes in advanced chronic liver disease (2). This is coherent with the clinical observation of thrombotic events occurring among cirrhotic patients, despite altered prothrombin- and partial thromboplastin-time, which are inadequate to evaluate the profound changes in balance between pro- and anti-coagulant factors alongside the disruption of liver function $(3,4)$. Moreover, plasma from patients with cirrhosis shows a pro-coagulant imbalance alongside disease severity, as expressed by the resistance to the action of thrombomodulin, a strong anti-coagulant factor (5). These pro-hemostatic properties are also associated with severe prognosis and probably concur in the worsening of liver function as consequence of liver microthrombosis and progressive parenchymal extinction $(6,7)$. As a matter of fact, in a cardinal randomized controlled trial by Villa et al., the use of enoxaparin was associated with better survival and less events of decompensation, along with portal vein thrombosis (PVT) prevention (8). Thus, the impact of all these changes in hemostasis on the development of PVT has been of great interest in recent years with important clinical meanings, due to the high frequency of PVT in the natural history of cirrhosis and the potential pathogenic role of hemostasis as mediator of liver damage. Moreover, PVT per se may lead to several complications like development or worsening of ascites and variceal bleeding and may compromise the viability of liver transplantation (9). Besides heparin and vitamin-K antagonists and, possibly, new direct anticoagulants (10), little is known about other pharmacological strategies for prevention and treatment of PVT (9). Therefore, understanding the mechanisms of thrombus formation in chronic liver disease is crucial, due to the fragile hemostatic profile described in cirrhosis.

We read with interest the recent paper by El-Makarem et al., which focuses on the potential contribution of inflammation in the pathophysiology of PVT (11). In this clinical study, authors analyzed several hemostatic parameters by comparing $\mathrm{HCV}$-positive cirrhotic patients with PVT $(\mathrm{n}=30)$, cirrhotic patients without PVT $(\mathrm{n}=35)$, non-cirrhotic patients with PVT $(n=15)$, and healthy controls $(n=15)$. All patients with PVT were not on anticoagulation at time of inclusion. First, they confirmed the futility of standard coagulation tests to evaluate the hemostatic balance in cirrhosis, indeed no INR differences were detected between PVT and non-PVT group. Second, they found reduced levels of pro-coagulant factor VII in cirrhosis, as expected for the advanced liver disease (1), and, in both cirrhoticand non-cirrhotic-PVT patients, found an increasing trend of FVII-AT complexes, which are considered markers of 
endogenous activated coagulation (12). To evaluate the potential role of inflammatory stimuli in PVT development, they focused on microparticles analysis, particularly on monocytic tissue factor (TF). Microparticles are extracellular vesicles extensively studied in hemostasis due to the capability to carry on their surface pro-coagulant molecules, like TF or negatively charged phospholipids. Their secretion is the consequence of several inflammatory stimuli like endotoxemia or cancer-related inflammation (13). They have been investigated in different clinical contexts and, recently, in the development of neoplastic PVT (14). In their study, El-Makarem et al. found an increase of monocytic-TF in both PVT-groups, without differences between non-cirrhotic $v s$.cirrhotic-PVT. This suggests an influence of such pro-hemostatic particles despite of liver function decline. Interestingly, together with portal vein diameter, monocytic-TF was independently associated with PVT, confirming a strong association of immuneinflammatory elements with thrombosis. Along with hemostatic parameters they found a reduction in HLADR expression in PVT-groups and increased levels of monocyte co-stimulatory molecule CD46 independently by the presence of cirrhosis. These indexes of profound immunological disturbance $(15,16)$ are coherent, although not specific, with the link between immune-system and hemostasis and warrant further investigations. The absence of differences between cirrhotic and non-cirrhotic PVT suggests the determinant role of immune system in the susceptibility to PVT whatever the degree of liver damage.

Globally, the results of this study are in line with a recent pathophysiological theory, by Bernardi et al., which considers the chronic activation of inflammatory pathways, due to the progressive increase of bacterial translocation from the gut, the main culprit of the detrimental progression of cirrhosis toward an end-stage liver disease (17). The novelty of the paper lies on bridging inflammation and hemostasis through microparticles, extensively studied in hematology and oncology fields but only at dawn in hepatology. The study is also on the same track as other several basic-science studies, which have enlightened the potential of hemostasis as effector of the innate immunesystem and, in turn, thrombosis as an overexpression of a natural defense system. The term "immuno-thrombosis" synthetizes these theories (18). As El-Makarem underlines, TF-bearing microparticles may represent the link between immune activation and thrombus formation as they may origin from monocytes, neutrophils, endothelial cells, which have in common a potential activation by endotoxemia (19). Unfortunately, authors did not measure any bacterial by-product to correlate with microparticles, therefore the pathogenic scenario of endotoxemia as trigger of inflammation, although biologically plausible, remains undemonstrated by this experience. In addition, the design of the study allows only to reveal a simple association between TF-bearing microparticles and PVT. A proper cause-effect relation would have been better addressed by a cohort study considering different levels of microparticles as risk factors for PVT, possibly with a dose-effect relation, or by an intervention neutralizing TF-bearing microparticles with an effect on the risk of PVT. This notwithstanding, the pathogenic caveat may be overcome by other similar articles in the field. At today, we have learnt that the procoagulant imbalance described in cirrhosis is sustained by high levels of factor VIII/protein C ratio and low levels of anticoagulants all correlating with the degree of plasma in vitro resistance to the action of thrombomodulin. These pro-hemostatic features are associated with worse prognosis, progression of the disease and translocation of bacterial products (7,20-22). A relevant weakness of the study by El-Makarem lies on the absence of measurement of these factors, as they are crucial players in the hemostatic balance, beside FVII (and FVII-AT complexes). Levels of FVIIAT complexes alone do not fully evaluate the hemostatic balance and therefore, the predominance of the immuneinflammatory component over the hypercoagulable state in the pathogenesis of PVT is an authors' hypothesis which needs further explorations. Moreover, along with hemostasis, hemodynamic alterations of cirrhosis participate to the pathologic milieu prone to thrombus formation. Altered venous flow in portal system is a crucial element independently associated to PVT development (23). Thus, endothelial damage with the release of microparticles, von Willebrand factor (VWF) and TF, together with prohemostatic proprieties of plasma and altered flow, all concur in the pathophysiology of cirrhosis and resemble the historical "Virchow's triad". This complex and intriguing interplay possibly clarifies the high prevalence of PVT in this population. These multiple interactions, ideally, offer many potential targets of therapy to prevent thrombus formation. In addition, recently, Tripodi et al. suggested that HCV-clearance could interfere with the pro-coagulant profile of plasma detected before antiviral treatment (24), but studies with larger cohorts are eagerly awaited to draw definitive conclusions on this topic. Notwithstanding, anticoagulation remains the cornerstone of PVT treatment, with beneficial risk-benefit ratio if the correct prophylaxis 
of variceal bleeding is provided (25).

In conclusion, new theories recognize inflammation as a crucial factor in disease progression due to the influence on several pro-hemostatic triggers, like TFbearing microparticles, with thrombus formation and PVT development in cirrhosis. The understanding of these proinflammatory, pro-hemostatic triggers may help to develop new diagnostic and therapeutic strategies to smother the pernicious impact of thrombosis on the natural history of cirrhosis.

\section{Acknowledgements}

Funding: This study was in part granted by the 'Fondo 5 Xmille per la Ricerca' (2011) from Italian Ministry of Health (Progetto: 5M-2017-2365131 - 5 per mille).

\section{Footnote}

Conflicts of Interest: The authors have no conflicts of interest to declare.

\section{References}

1. Tripodi A, Primignani M, Mannucci PM, et al. Changing Concepts of Cirrhotic Coagulopathy. Am J Gastroenterol 2017;112:274-81.

2. Tripodi A, Salerno F, Chantarangkul V, et al. Evidence of normal thrombin generation in cirrhosis despite abnormal conventional coagulation tests. Hepatology 2005;41:553-8.

3. Northup PG, McMahon MM, Ruhl AP, et al. Coagulopathy does not fully protect hospitalized cirrhosis patients from peripheral venous thromboembolism. Am J Gastroenterol 2006;101:1524-8.

4. Tripodi A, Chantarangkul V, Primignani M, et al. The international normalized ratio calibrated for cirrhosis (INR liver) normalizes prothrombin time results for model for end-stage liver disease calculation. Hepatology 2007;46:520-7.

5. Tripodi A, Primignani M, Lemma L, et al. Evidence that low protein $\mathrm{C}$ contributes to the procoagulant imbalance in cirrhosis. J Hepatol 2013;59:265-70.

6. Wanless IR, Wong F, Blendis LM, et al. Hepatic and portal vein thrombosis in cirrhosis: possible role in development of parenchymal extinction and portal hypertension. Hepatology 1995;21:1238-47.

7. La Mura V, Tripodi A, Tosetti G, et al. Resistance to thrombomodulin is associated with de novo portal vein thrombosis and low survival in patients with cirrhosis. Liver Int 2016;36:1322-30.

8. Villa E, Cammà C, Marietta $M$, et al. Enoxaparin prevents portal vein thrombosis and liver decompensation in patients with advanced cirrhosis. Gastroenterology 2012;143:1253-60.e1-4.

9. Primignani M, Tosetti G, La Mura V. Therapeutic and clinical aspects of portal vein thrombosis in patients with cirrhosis. World J Hepatol 2015;7:2906-12.

10. De Gottardi A, Trebicka J, Klinger C, et al. Antithrombotic treatment with direct-acting oral anticoagulants in patients with splanchnic vein thrombosis and cirrhosis. Liver Int 2017;37:694-9.

11. Abu El-Makarem MA, El-Akad AF, Elian MM, et al. Nonneoplastic Portal Vein Thrombosis in HCV Cirrhosis Patients: Is it an Immuno-Inflammatory Disorder? Ann Hepatol 2017;16:574-83.

12. Spiezia L, Rossetto V, Campello E, et al. Factor VIIaantithrombin complexes in patients with arterial and venous thrombosis. Thromb Haemost 2010;103:1188-92.

13. Barteneva NS, Fasler-Kan E, Bernimoulin M, et al. Circulating microparticles: square the circle. BMC Cell Biol 2013;14:23.

14. Campello E, Zanetto A, Spiezia L, et al. Hypercoagulability detected by circulating microparticles in patients with hepatocellular carcinoma and cirrhosis. Thromb Res 2016;143:118-21.

15. Cajander S, Bäckman A, Tina E, et al. Preliminary results in quantitation of HLA-DRA by real-time PCR: a promising approach to identify immunosuppression in sepsis. Crit Care 2013;17:R223.

16. Liszewski MK, Atkinson JP. Complement regulator CD46: genetic variants and disease associations. Hum Genomics 2015;9:7.

17. Bernardi M, Moreau R, Angeli P, et al. Mechanisms of decompensation and organ failure in cirrhosis: From peripheral arterial vasodilation to systemic inflammation hypothesis. J Hepatol 2015;63:1272-84.

18. Engelmann B, Massberg S. Thrombosis as an intravascular effector of innate immunity. Nat Rev Immunol 2013;13:34-45.

19. Shantsila E, Lip GY. The role of monocytes in thrombotic disorders. Insights from tissue factor, monocyte-platelet aggregates and novel mechanisms. Thromb Haemost 2009;102:916-24.

20. Ferro D, Quintarelli C, Lattuada A, et al. High plasma levels of von Willebrand factor as a marker of endothelial perturbation in cirrhosis: relationship to endotoxemia. 
Hepatology 1996;23:1377-83.

21. La Mura V, Reverter JC, Flores-Arroyo A, et al. Von Willebrand factor levels predict clinical outcome in patients with cirrhosis and portal hypertension. Gut 2011;60:1133-8.

22. Kalambokis GN, Oikonomou A, Christou L, et al. von Willebrand factor and procoagulant imbalance predict outcome in patients with cirrhosis and thrombocytopenia. J Hepatol 2016;65:921-8.

23. Zocco MA, Di Stasio E, De Cristofaro R, et al.

Thrombotic risk factors in patients with liver cirrhosis:

doi: 10.21037/amj.2017.09.12

Cite this article as: Bitto N, La Mura V. Non-neoplastic portal vein thrombosis in $\mathrm{HCV}$ cirrhosis: the weight of inflammation on a fragile hemostatic balance. AME Med J 2017;2:150. correlation with MELD scoring system and portal vein thrombosis development. J Hepatol 2009;51:682-9.

24. Tripodi A, D'Ambrosio R, Padovan L, et al. Evaluation of coagulation during treatment with directly acting antivirals in patients with hepatitis $\mathrm{C}$ virus related cirrhosis. Liver Int 2017;37:1295-303.

25. Under the auspices of the Italian Association for the Study of Liver Diseases (AISF) and the Italian Society of Internal Medicine (SIMI). Hemostatic balance in patients with liver cirrhosis: Report of a consensus conference. Dig Liver Dis 2016;48:455-67. 